Daniel Amaral de M. Rocha

Combinando metaeurísticas com resolvedores MIP, com aplicações ao Generalized Assignment Problem (GAP)

Dissertação apresentada como requisito parcial para obtenção do grau de Mestre pelo Programa de Pós-graduação em Informática do Departamento de Informática da PUC-Rio

Orientador: Prof. Marcus V. S. Poggi de Aragão 




Daniel Amaral de M. Rocha

\section{Combinando metaeurísticas com resolvedores \\ MIP, com aplicações ao Generalized Assignment Problem (GAP)}

Dissertação apresentada como requisito parcial para obtenção do grau de Mestre pelo Programa de Pós-graduação em Informática do Departamento de Informática do Centro Técnico Científico da PUC-Rio. Aprovada pela Comissão Examinadora abaixo assinada.

Prof. Marcus V. S. Poggi de Aragão

Orientador

Departamento de Informática — PUC-Rio

Prof. Eduardo Laber

Departamento de Informática - PUC-Rio

Prof. Lorenza Leao Oliveira Moreno

Departamento de Informática - PUC-Rio

Prof. José Eugenio Leal

Coordenador Setorial do Centro Técnico Científico - PUC-Rio

Rio de Janeiro, 11 de Agosto de 2009 
Todos os direitos reservados. É proibida a reprodução total ou parcial do trabalho sem autorização da universidade, do autor e do orientador.

Daniel Amaral de M. Rocha

BSc. em Ciências da Computação pela Universidade Federal do Rio Grande do Norte em 2006, entrou no programa de pós-graduação do Departamento de Informática da PUC-Rio em 2007. Participou durante alguns meses, como pesquisador, do projeto de otimização de rotas de navios (PRONAV) da Petrobrás. Ao fim de 2007, mudou-se para Nova York, onde vive desde então.

Ficha Catalográfica

Rocha, Daniel Amaral de Medeiros

Combinando metaeurísticas com resolvedores MIP, com aplicações ao Generalized Assignment Problem (GAP) / Daniel Amaral de M. Rocha; orientador: Marcus V. S. Poggi de Aragão. - Rio de Janeiro : PUC-Rio, Departamento de Informática, 2009.

v., 71 f: il. ; $29,7 \mathrm{~cm}$

1. Dissertação (mestrado) - Pontifícia Universidade Católica do Rio de Janeiro, Departamento de Informática.

Inclui referências bibliográficas.

1. Informática - Tese. 2. Metaeurísticas. 3. Path Relinking. 4. Vizinhança elipsoidal. 5. Otimização Combinatória. 6. Programação Inteira Mista. 7. Problema de Alocação Generalizado. I. Aragão, Marcus V. S. Poggi de. II. Pontifícia Universidade Católica do Rio de Janeiro. Departamento de Informática. III. Título. 


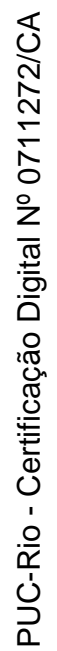

Para Thaisa 


\section{Agradecimentos}

Primeiramente não posso deixar de agradecer ao apoio dado por minha esposa. Thaisa, minha inspiração e força de vontade. Obrigado por todas as tardes em casa me apoiando, revisando o texto, me acalmando com uma xicara de café (ou um cafuné), acordada até de madrugada (ou, pior, acordando cedo), todos aqueles finais de semana trabalhando o dia todo (e a noite também). Sem você, sua força e seu apoio constantes, esse trabalho simplesmente não teria acontecido. Obrigado.

A Marcus Poggi, por todos os conselhos, direções, e-mails e reuniões. Por ter sido calmo quando eu sumia e tranquilo quando eu me desesperava. Por ter respondido todas minhas mensagens, mesmo as que tinham dezenas de perguntas diferentes. Não consigo imaginar um orientador melhor - além de tudo, ele ainda é tricolor.

A minha família, que sempre me mandou apoio apesar da distância. Em especial a minha mãe, que me ajudou a escolher esse caminho quando eu estava saindo da graduação e cujo incentivo foi fundamental pra que eu continuasse trabalhando. Lembro de nossas conversas no fim de 2006 sobre minha ida pro mestrado - seu apoio naquele momento foi muito importante e, apesar de tudo, não me arrependo em nada das decisões que tomamos.

Finalmente, agradeço a Família Glória, com quem convivi durante o ano mais tumultuado da minha vida e que sempre estiveram (e sempre estarão) comigo. Raoni, Aurélia, César, o ano que passei vivendo com vocês foi incrível e, sem sua companhia, meu mestrado teria se encerrado antes de começar. A amizade de vocês é, sem dúvida, o melhor resultado da minha pós-graduação. 


\section{Resumo}

Rocha, Daniel Amaral de Medeiros; Aragão, Marcus V. S. Poggi de. Combinando metaeurísticas com resolvedores MIP, com aplicações ao Generalized Assignment Problem (GAP). Rio de Janeiro, 2009. 71p. Dissertação de Mestrado — Departamento de Informática, Pontifícia Universidade Católica do Rio de Janeiro.

Métodos que combinam estratégias normalmente encontradas em algoritmos metaeurísticos com técnicas para resolver problemas de programação inteira mista (MIP) têm apresentado ótimos resultados nos últimos anos. Este trabalho propõe dois novos algoritmos nessa linha: um algoritmo baseado na metaeurística Path Relink (PR) e um simples algoritmo que faz pós-processamento nas soluções encontradas pelo resolvedor MIP. Os dois algoritmos utilizam um novo tipo de vizinhança, chamada de vizinhaça elipsoidal, que possui fortes semelhanças com as técnicas de relinking de algoritmos PR e que neste trabalho é generalizada e extendida para múltiplas soluções. O problema generalizado de alocação (GAP) é usado para os experimentos. São testados também um resolvedor MIP puro (ILOG CPLEX versão 11) e um algoritmo branch and price que utiliza as heurísticas RINS e guided dives. Os algoritmos testados são comparados entre si e com heurísticas específicas para o GAP. Os resultados são satisfatórios e indicam que as vizinhanças elipsoidais conseguem frequentemente melhorar as soluções encontradas pelo resolvedor MIP, encontrando a melhor solução para algumas instâncias.

\section{Palavras-chave}

Metaeurísticas. Path Relinking. Vizinhança elipsoidal. Otimização Combinatória. Programação Inteira Mista. Problema de Alocação Generalizado. 


\section{Abstract}

Rocha, Daniel Amaral de Medeiros; Aragão, Marcus V. S. Poggi de. Combining metaheuristics with MP solvers, with applications to the Generalized Assignment Problem (GAP). Rio de Janeiro, 2009. 71p. MSc. Dissertation — Departamento de Informática, Pontifícia Universidade Católica do Rio de Janeiro.

Methods that mix strategies usually found in metaheuristic algorithms with techniques to solve mixed integer programming problems (MIPs) have had great results over the past few years. This work proposes two new algorithms in this philosophy: one is based on the Path Relink (PR) metaheuristic, while the other one is a simple algorithm that does post-processing in the solutions found by the MIP solver. Both algorithms use a new neighborhood structure, called ellipsoidal neighborhood, that has strong resemblances with the relinking step from PR algorithms and that, in this work, is generalized and extended for multiple solutions. The generalized assignment problem (GAP) is used for the computational experiments. Also tested are a MIP solver (ILOG CPLEX version 11) and a branch and price algorithm that uses the RINS and guided dives heuristics. The tested algorithms are compared among themselves and with GAP-specific heuristics. The results are satisfatory and show that the ellipsoidal neighborhoods can frequently improve the solutions found by the MIP solver, even finding the best result for some instances.

\section{Keywords}

Metaheuristics. Path Relinking. Ellipsoidal neighborhood. Combinatorial Optimization. Mixed Integer Programming. Generalized Assignment Problem. 


\section{Sumário}

1 Introdução 11

$\begin{array}{lll}1.1 & \text { Organização deste trabalho } & 12\end{array}$

2 Heurísticas e métodos aproximados $\quad 14$

2.1 Busca local 14

$\begin{array}{lll}2.2 & \text { Metaeurísticas } & 15\end{array}$

$\begin{array}{lll}2.3 \text { Dificuldades } & 18\end{array}$

3 Programação Inteira e resolvedores MIP $\quad 20$

3.1 Resolvendo MIPs 20

$\begin{array}{ll}3.2 & \text { Dificuldades } \\ & 22\end{array}$

4 Combinando heurísticas com resolvedores MIP 23

4.1 Taxonomia 23

4.2 Local Branching (LB) 25

4.3 Relaxation Induced Neighborhood Search (RINS) 26

4.4 Variable Neighborhood Search (VNS) 28

5 Vizinhança elipsoidal $\quad 31$

5.1 Múltiplas soluções $\quad 33$

5.2 Paralelo com Relinking 36

$6 \quad$ Generalized Assignment Problem (GAP) $\quad 37$

6.1 Formulação clássica 38

6.2 Formulação com número exponencial de colunas (geração de colunas) 39

6.3 Revisão da literatura do problema 40

6.4 Instâncias 43

7 Algoritmos $\quad 45$

7.1 CPLEX 45

7.2 Pós-processamento com vizinhanças elipsoidais (PostProcessing) 46

7.3 Variable Neighborhood Seach Branching (VNSBra) 48

7.4 Path Relink com vizinhança elipsoidal (PathRelinkMIP) 48

7.5 Stabilized branch-and-price com RINS e guided dives (BPRINS) 50

$\begin{array}{ll}7.6 & \text { TSEC e PREC } \\ \end{array}$

$8 \quad$ Experimentos e Resultados $\quad 52$

8.1 Metodologia $\quad 52$

8.2 CPLEX 53

8.3 PostProcessing 56

8.4 VNSBra $\quad 56$

8.5 PathRelinkMIP 58

8.6 Algoritmos MIP: PathRelinkMIP vs. VNSBra vs. CPLEX 59

8.7 BPRINS 61

8.8 Algoritmos MIP vs. BPRINS vs. metaeurísticas 63 
Referências Bibliográficas 


\section{Lista de tabelas}

8.1 Resultados do algoritmo CPLEX para as instâncias classe C 54

8.2 Resultados do algoritmo CPLEX para as instâncias classe D 54

8.3 Resultados do algoritmo CPLEX para as instâncias classe E 55

8.4 Comparação dos algoritmos PostProcessing e CPLEX 57

8.5 Resultados do algoritmo VNSBra 58

8.6 Resultados do algoritmo PathRelinkMIP 60

8.7 Comparação dos algoritmos PathRelinkMIP, VNSBra e CPLEX 62

8.8 Resultados do algoritmo BPRINS, comparados com CPLEX 63

8.9 Comparação de todos os algoritmos 65 